H. Neuhauser A. Dippelhofer H. Hölling

\section{Befragung zur körperlichen Gesundheit im Rahmen des Kinder- und Jugendgesundheitssurveys}

Questionnaire and Interview on Physical Health in the National Health Interview and Examination Survey for Children and Adolescents

\section{Zusammenfassung}

Die körperliche Gesundheit ist auch jenseits einer nur krankheitsorientierten Betrachtung eine wichtige Komponente der Gesundheit. Differenzierte, umfassende und repräsentative Daten über die körperliche Gesundheit von Kindern und Jugendlichen in Deutschland fehlen jedoch bislang. Sie sollen im Rahmen des Kinder- und Jugendgesundheitssurveys erstmals erhoben werden. Dies schließt Informationen zu Schwangerschaft, Geburt und Entwicklung, subjektivem Gesundheitszustand, körperlichem Wohlbefinden, akuten und chronischen Krankheiten, angeborenen Fehlbildungen, Verletzungen, Beschwerden, Behinderungen und Schmerzen ein. Dadurch, dass auch detaillierte Fragen zu Gesundheitsrisiken, Unfällen, Ernährung, körperlicher Aktivität, präventivem Verhalten, Lebensqualität, psychischer Gesundheit, Umweltbedingungen, soziodemografischen Merkmalen und Inanspruchnahme medizinischer Leistungen gestellt werden, können zahlreiche komplexe Zusammenhänge zur körperlichen Gesundheit von Kindern und Jugendlichen in Deutschland erstmals umfassend untersucht werden.

Im Folgenden werden die Fragen zur körperlichen Gesundheit aus den Fragebogen und dem ärztlichen Interview im Überblick dargestellt. Dabei werden der inhaltliche und methodische Hintergrund und die Erfahrungen aus einem Pretest kurz skizziert, die zur Auswahl der Fragen und zu ihrer Zuordnung zu den Befragungsinstrumenten beigetragen haben.

\section{Schlüsselwörter}

Kinder- und Jugendgesundheitssurvey • körperliche Gesundheit · Fragebogen · CAPI $\cdot$ Krankheiten

\section{Abstract}

Physical health is an important dimension within even the broadest concept of health. However, comprehensive and representative data on the physical health of children and adolescents in Germany are so far lacking. In the Health Survey for Children and Adolescents information will be collected on various aspects of physical health: pregnancy, birth, child development, selfrated health, physical well-being, acute and chronic disorders, congenital malformations, injuries, symptoms and complaints, disability and pain. It will be possible to analyse various determinants and consequences of physical health of children in Germany since data will also be collected on health risks, nutrition, physical activity, preventive behavior, quality of life, mental health, environmental aspects, socioeconomic and demographic characteristics and use of health services. This paper summarises the items on physical health assessed in the questionnaires and the medical interview, outlines the theoretical background for some methodological issues and reports conclusions from the survey pretest.

\section{Key words}

Health survey for children and adolescents - physical health · questionnaire $\cdot$ CAPI $\cdot$ diseases 


\section{Relevanz und Datenlage}

Das Krankheitsgeschehen im Kindes- und Jugendalter hat in den letzten Jahrzehnten erhebliche Veränderungen erfahren. Diese sind teilweise positiv, wie z. B. das deutliche Absinken der Kinder- und Säuglingssterblichkeit, der Rückgang von Infektionskrankheiten wie Tuberkulose, Pocken, Poliomyelitis, Scharlach, Diphterie, Meningitis und Wundstarrkrampf, oder die verbesserte Therapie und dadurch erhöhte Lebenserwartung von Kindern mit chronischen Krankheiten wie z. B. zystischer Fibrose, angeborenen Herzfehlern oder Leukämie. Dem stehen jedoch deutliche Hinweise für eine besorgniserregende und beobachtungsbedürftige Entwicklung gegenüber: die Zunahme von chronischen Krankheiten im Kindes- und Jugendalter.

Krankheiten kommen bei Kindern und Jugendlichen insgesamt seltener vor als im Erwachsenenalter. Sie sind in dieser Lebensphase aber von besonderer Bedeutung, weil sie die Entwicklung des Kindes nachhaltig beeinträchtigen können und das Auftreten chronischer Erkrankungen im Erwachsenenalter mitbestimmen [Gillman 2002, Kuh, Ben-Shlomo 1997].

Repräsentative Studien zur Verbreitung akuter und chronischer Erkrankungen im Kindes- und Jugendalter in Deutschland existieren bislang nur für einige Erkrankungen und auch nur für ausgewählte Altersgruppen [Hoepner-Stamos 1995, Kolip et al. 1995]. Zusammenhänge zu Einflussfaktoren können oft nicht hergestellt werden, weil zu diesen keine Informationen erhoben wurden. Routinedaten können helfen, einzelne Aspekte zu beleuchten [Bundeszentrale für gesundheitliche Aufklärung 1998], viele Fragen bleiben aber offen, weil die vorhandenen Datenquellen nicht miteinander verknüpfbar sind. Ergebnisse aus internationalen Studien können nicht ohne weiteres auf Deutschland übertragen werden, da in den wenigen vergleichenden Arbeiten zum Teil erhebliche Unterschiede zwischen Ländern gefunden wurden [Beasley et al. 2000, Silink 2002, Currie et al. 2000].

Wie viele Kinder in Deutschland insgesamt chronisch krank sind, lässt sich auf der Grundlage der verfügbaren Daten bislang nicht bestimmen. Im Mikrozensus wurde 1974 bis 1982 im Rahmen der Fragen zur Gesundheit auch eine Frage zum Vorliegen von chronischen Erkrankungen gestellt. Diese Frage wurde 1982 für 1,17\% der Kinder und Jugendlichen bis 15 Jahre bejaht [Statistisches Bundesamt 1984] - allerdings muss diese Prävalenz aufgrund methodischer Besonderheiten stark angezweifelt werden. An den Befragungen zu chronischen Krankheiten, die in den USA durchgeführt wurden, lässt sich zeigen, wie stark Prävalenzschätzungen von der methodischen Vorgehensweise, vor allem der Definition des Begriffes „chronische Krankheit“ abhängen. So ergab die Befragung der Eltern von über 18000 Kindern im Rahmen des National Health Interview Surveys 1988, dass 31\% der US-amerikanischen Kinder chronisch krank sind [Newascheck, Taylor 1992]. Dieser Zahl liegt eine umfassende Liste von einzeln abgefragten Krankheiten zugrunde sowie die Angabe jeder anderen Erkrankung, die zum Interviewzeitpunkt bereits länger als drei Monate andauerte. $\mathrm{Zu}$ den häufigsten chronischen Erkrankungen zählten Asthma mit 4,3\%, andere allergische Atemwegserkrankungen mit 9,7\% und wiederholte Ohrentzündungen mit 8,4\%. Unter Hinzuziehung von Fragen zu Beeinträchtigung und Aktivitätseinschränkungen durch chronische Krank- heiten konnte diese hohe Zahl von chronischen Erkrankungen jedoch differenzierter betrachtet werden: Nur 2\% aller Kinder waren durch chronische Krankheiten schwer betroffen, 9\% mäßig stark und 20\% leicht. Im Zeitverlauf zeigen die Ergebnisse des National Health Interview Surveys einen alarmierenderen Trend: 1995 war die Beeinträchtigung durch chronische Erkrankungen bei Kindern mehr als doppelt so hoch wie 25 Jahre zuvor, speziell durch Asthma sogar dreimal höher [Newacheck, Halfon 2000].

Neben Erkrankungen sind auch Beschwerden, Behinderungen und Entwicklungsmerkmale für die Charakterisierung der körperlichen Gesundheit von Bedeutung. Sowohl pränatale als auch perinatale Faktoren können die Gesundheit von Kindern beeinflussen, daher sollten auch Merkmale der Schwangerschaft und Geburt betrachtet werden [Barker et al. 1993, Whitaker, Dietz 1998, Blake 2000, Dörner, Plagemann 1994]. Schmerzen sind ein erschreckend häufiges, aber noch wenig untersuchtes Problem bei Kindern und Jugendlichen [Perquin et al. 2000a]. Sie beeinflussen die Lebensqualität und die körperliche und psychische Entwicklung und sind ein wichtiger Faktor für die Inanspruchnahme von Gesundheitsleistungen. Unfälle sind in Europa gegenwärtig die häufigste Todesursache bei 5- bis 19-Jährigen [The European Health Report 2002]. Ein besseres Verständnis ihrer bestimmenden Faktoren ist eine Voraussetzung für die Verbesserung von Präventionsstrategien. Schließlich belegen Untersuchungen eindrucksvoll, dass die Selbsteinschätzung der Gesundheit auch bei umfassender Datenerhebung zu Krankheiten, Beschwerden, Risikofaktoren, demographischen und sozioökonomischen Variablen einen unabhängigen Prädiktor für Mortalität und Morbidität [Idler, Benyamini 1997, Kaplan et al. 1996] darstellt.

\section{Auswahl der Fragen und Zuordnung zu den Befragungsinstrumenten}

Fragen zur körperlichen Gesundheit sind in den Eltern-Fragebogen (für die Altersstufen 0 bis 2, 3 bis 6,7 bis 10,11 bis 13,14 bis 17 Jahre), den Jugendlichen-Fragebogen (für die Altersstufen 11 bis 13 und 14 bis 17 Jahre) und in dem strukturierten computergestützten ärztlichen Interview enthalten. Die Fragen betreffen folgende Aspekte der körperlichen Gesundheit von Kindern und Jugendlichen:

- akute und chronische Krankheiten, angeborene Fehlbildungen, Operationen

- Schwangerschaft und Geburt

- Entwicklung

- Behinderungen

- Beschwerden

- körperliches Wohlbefinden

- subjektiver Gesundheitszustand

- Schmerzen

- Unfälle

- Impfungen

- Medikamentenkonsum

Kriterien für die Auswahl von Erkrankungen, die im Survey erfragt werden, waren die Prävalenz, Hinweise für Über-, Unterund Fehlversorgung, die gruppenspezifische Bedeutung (z. B. be- 
stimmte Altersgruppen), die besondere bevölkerungsbezogene Dynamik (Veränderung der Prävalenz, Risiken, Inanspruchnahme), die Beeinflussbarkeit, die gesetzlichen und politischen Rahmenbedingungen sowie die bisherige Verfügbarkeit von Daten.

Die Länge der Fragebogen und des ärztlichen Interviews, die einen direkten Einfluss auf die Mitarbeit der Befragten und damit auf die Validität der erhobenen Daten hat, war ein wichtiger limitierender Faktor bei der Auswahl der Items.

Die Fragen wurden nach methodischen und inhaltlichen Gesichtspunkten den zwei Befragungsinstrumenten (Fragebogen und ärztliches Interview) zugewiesen. Speziell für die Erfassung der Krankheiten galt es dabei einige grundsätzliche Fragen zu beantworten: Wie wird das Vorliegen einer Erkrankung operationalisiert? Im Rahmen methodischer Begleitstudien zum National Health Interview Survey in den USA konnte gezeigt werden, dass für die gleiche Studienpopulation verschiedene Erkrankungsprävalenzen ermittelt werden können, je nachdem ob 1 . die Betroffenen selbst (bzw. bei Kindern die Eltern) nach dem Vorliegen einer Erkrankung gefragt werden, ob 2. die Diagnosen aus den Krankenakten oder durch Befragung der behandelnden Ärzte erfasst werden oder ob 3. Ärzte im Rahmen eines Surveys nach definierten Kriterien Diagnosen stellen und dafür eine gezielte Anamnese erheben, eine körperliche Untersuchung und ggf. Labor- oder apparative Zusatzuntersuchungen durchführen [Gordon 1967, Miller 2001]. Diese dritte Möglichkeit der standardisierten Survey-Diagnosen verspricht einen hohen Grad an Validität und Reliabilität, ist jedoch aufgrund des zeitlichen und infrastrukturellen Aufwands solchen Surveys vorbehalten, die nur eine oder einige wenige Erkrankungen untersuchen. Die Erfassung der Diagnosen aus den Krankenakten oder durch Befragung der behandelnden Ärzte/Ärztinnen ist aufgrund des fraktionierten
Versorgungsgeschehens nicht praktikabel, wenn möglichst alle Erkrankungen, die ein Kind jemals hatte, erfasst werden sollen. Die Befragung der Eltern bzw. Jugendlichen bietet die Möglichkeit, auch Krankheiten zu erfassen, die ärztlich nicht oder noch nicht diagnostiziert wurden. Allerdings haben die so erfassten Prävalenzen zwei komplexe Komponenten: ärztlich gestellte Diagnosen, die verstanden, erinnert und wiedergegeben werden müssen, und Eigendiagnosen, in denen sich wandelnde und individuell differierende Laienkonzepte von Krankheiten wiederfinden.

Für ältere Kinder und Jugendliche ergibt sich schließlich auch noch die Frage: Ab welchem Alter können oder sollen Kinder bzw. Jugendliche selbst befragt werden? Untersuchungen zeigen, dass die Reliabilität der Antworten von Kindern und Jugendlichen zu Gesundheitsfragen umso geringer ist, je jünger die Kinder sind [Zill 2001]. Die Beantwortung von Fragen zu Krankheiten setzt ein ausreichendes Verständnis komplexer Krankheitskonzepte voraus. Je länger Krankheiten zurückliegen, desto jünger waren die Kinder, als sie davon betroffen waren. Dies erhöht das Problem des Erinnerungsbias erheblich. Insgesamt sind Fragen zu Wohlbefinden und Beschwerden zum Interviewzeitpunkt für Kinder und Jugendliche wesentlich leichter zu beantworten als Fragen zu Krankheiten, speziell zu vergangenen Krankheiten.

Für die Erfassung der körperlichen Gesundheit werden sich im Kinder- und Jugendsurvey vier verschiedene Ansätze ergänzen: Selbstausfüllfragebogen für Eltern, Selbstausfüllfragebogen für Kinder und Jugendliche ab elf Jahren, ein ärztliches Interview und ein Untersuchungsteil (Tab. 2).

Im ärztlichen Interview werden ärztliche Diagnosen (vor allem von chronischen Erkrankungen), die bis zum Interviewzeitpunkt gestellt wurden, erfasst. Die Fragen haben das Format: „Hat ein

Tab. 1 Fragebogen und ärztliches Interview: Krankheiten

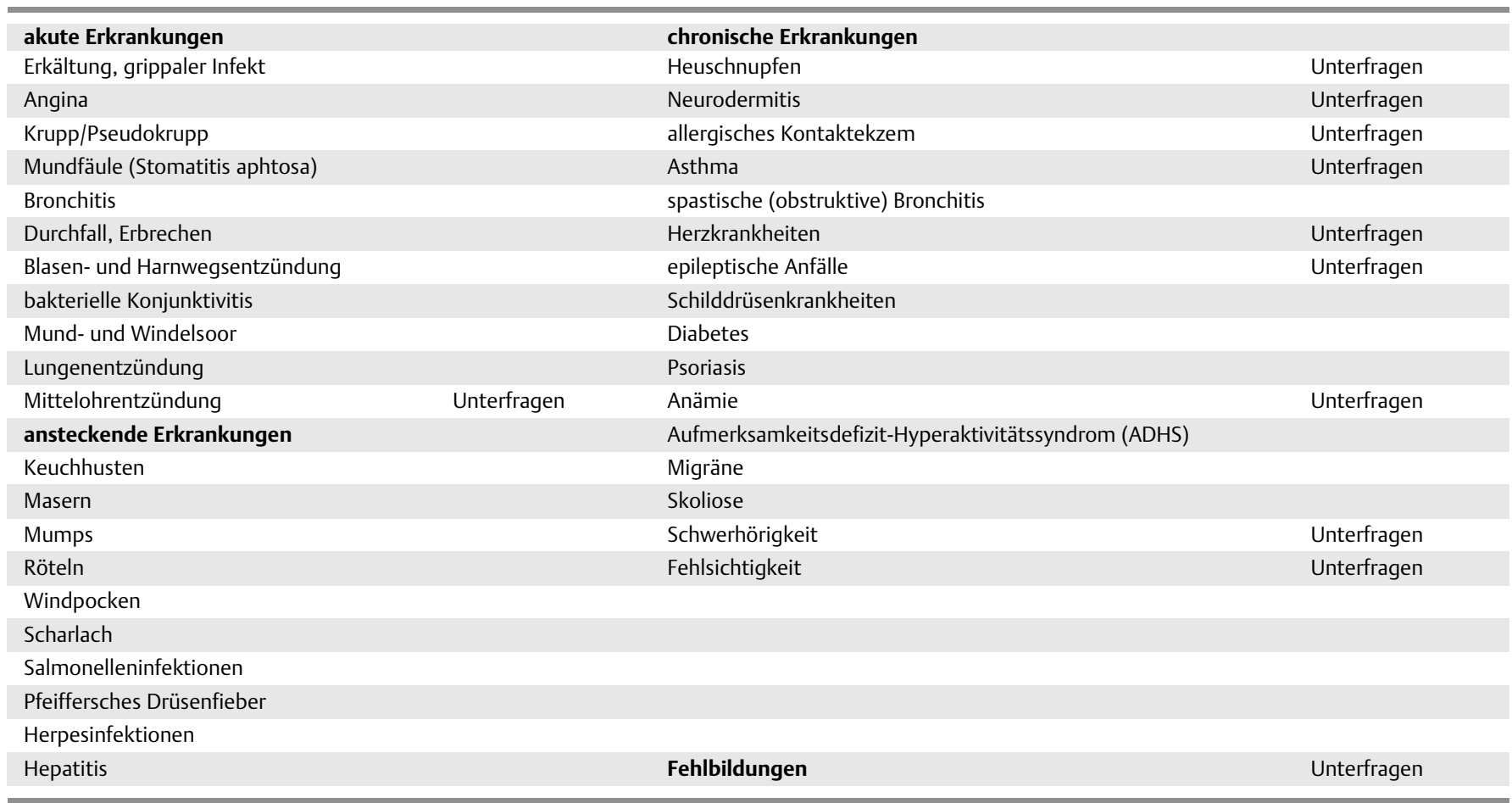


Tab. 2 Erfassung der körperlichen Gesundheit im Kinder- und Jugendsurvey

\begin{tabular}{|c|c|c|c|}
\hline Fragebogen (Eltern) & Fragebogen (Kinder und Jugendliche) & ärztliches Interview & $\begin{array}{l}\text { körperliche Untersuchung, apparative } \\
\text { Zusatzuntersuchungen und Labor- } \\
\text { diagnostik }\end{array}$ \\
\hline $\begin{array}{l}\text { körperliches Wohlbefinden } \\
\text { Beschwerden } \\
\text { körperliche Leistungsfähigkeit } \\
\text { Schmerzen } \\
\text { subjektive Gesundheit } \\
\text { akute Erkrankungen } \\
\text { chronische Erkrankungen } \\
\text { Schwangerschaft und Geburt } \\
\text { körperliche Entwicklung } \\
\text { Behinderungen } \\
\text { Unfälle }\end{array}$ & $\begin{array}{l}\text { ab } 11 \text { Jahren: } \\
\text { körperliches Wohlbefinden } \\
\text { Beschwerden } \\
\text { körperliche Leistungsfähigkeit } \\
\text { Schmerzen } \\
\text { ab } 14 \text { Jahren: } \\
\text { subjektive Gesundheit } \\
\text { akute Erkrankungen } \\
\text { (einige) chronische Erkrankungen }\end{array}$ & $\begin{array}{l}\text { ärztlich diagnostizierte Krankheiten } \\
\text { Unterfragen zu Krankheiten } \\
\text { Medikamentenkonsum } \\
\text { Impfungen }\end{array}$ & $\begin{array}{l}\text { ausgewählte objektivierbare Merkmale } \\
\text { der körperlichen Gesundheit }\end{array}$ \\
\hline
\end{tabular}

Arzt jemals bei Ihrem Kind die Krankheit X festgestellt?“ Die interviewenden Ärzte können durch Nachfragen die Plausibilität der Angaben prüfen und helfen, die nosologisch richtige Bezeichnung zu finden. Zusätzlich werden das Alter bei erstmaliger Feststellung der Erkrankung, das Auftreten in den letzten 12 Monaten und in den letzten 4 Wochen erfragt. Neben der Prävalenz werden für einige - vorwiegend chronische - Erkrankungen durch Unterfragen Daten zu zusätzlichen Aspekten erhoben, z.B. zu speziellen Krankheitsmerkmalen, Krankheitsfolgen, durchgeführter Diagnostik und Therapie, Versorgung und Inanspruchnahme. Über offene Fragen und Freitextfelder werden sowohl in den Fragebogen als auch im ärztlichen Interview zusätzliche Erkrankungen erfasst.

Die Erfassung der Medikamenteneinnahme und des Impfstatus im ärztlichen Interview sowie der Untersuchungsteil werden an einer anderen Stelle detailliert beschrieben [Knopf et al. 2002].

Kinder mit besonderen Bedürfnissen hinsichtlich ihrer Gesundheitsversorgung sollen auch losgelöst von spezifischen Diagnosen identifiziert werden. Art und Umfang von amtlich anerkannten Behinderungen werden erfragt und darüber hinaus wird ein spezielles Screening-Instrument (Children with Special Health Care Needs oder CSHCN Screener) [Bethell et al. 2002], bestehend aus fünf Fragen mit jeweils zwei Unterfragen, eingesetzt. Dabei wird gefragt, ob das Kind vom Arzt verschriebene Medikamente einnimmt, ob es überdurchschnittlich viel medizinische, psychosoziale oder pädagogische Unterstützung braucht, Einschränkungen oder Behinderungen im Alltag hat, eine spezielle Therapie benötigt oder bekommt und ob es emotionale, entwicklungs- oder verhaltensbezogene Probleme hat, für die es eine Behandlung bzw. Beratung benötigt oder bekommt.

Zu dem Themenbereich Schwangerschaft, Geburt und Entwicklung werden diverse Aspekte des Schwangerschaftsverlaufs, Geburt, perinatale Probleme, Entwicklungsmerkmale und Entwicklungsverlauf der Kinder erfragt. Unfällen und Vergiftungen ist eine Reihe von Fragen gewidmet. Erfragt werden nicht nur Verletzungen und deren Behandlung, sondern auch Art, Ort, Beteiligung besonderer Umstände oder Gegenstände sowie das Präventionsbzw. Risikoverhalten. Basierend auf einem niederländischen Instrument [Perquin et al. 2000b] wird ein Schmerzfragebogen für Kinder eingesetzt, der Fragen zur Prävalenz von Schmerzen, zur Lokalisation, Dauer und Intensität beinhaltet. Die Erfassung von
Schmerzen im Kinder- und Jugendsurvey wird detailliert an einer anderen Stelle beschrieben [Roth-Isigkeit et al. 2002]. Aber auch andere standardisierte Instrumente, die in die Fragebogen integriert wurden, enthalten Fragen zur körperlichen Gesundheit: Im KINDL-Fragebogen zur Erfassung der gesundheitsbezogenen Lebensqualität, den alle Eltern, aber auch Kinder und Jugendliche ab 11 Jahren beantworten, finden sich Fragen zum körperlichen Wohlbefinden [Ravens-Sieberer, Bullinger 1998], und im Rahmen des Strengths and Difficulties Questionnaire (SDQ) [Goodman 2001], einem Instrument zur Untersuchung psychischer Auffälligkeiten im Kindesalter, der in den Elternfragebogen enthalten ist, wird nach körperlichen Beschwerden (nämlich nach häufigem Klagen über Kopfschmerzen, Bauchschmerzen oder Übelkeit) gefragt.

Der allgemeine Gesundheitszustand wird auf einer Skala mit fünf Kategorien (von sehr gut bis sehr schlecht) eingeschätzt. In Hinblick auf eine internationale Vergleichbarkeit wurde die von der WHO empfohlene Formulierung gewählt [De Bruin et al. 1996].

Die Angaben zur körperlichen Gesundheit sollen sowohl mit soziodemographischen Faktoren wie beispielsweise Alter, Geschlecht, Sozialschicht oder Wohnort, als auch mit Indikatoren für die psychische Gesundheit und Lebensqualität oder Belastungen in der Umwelt in Beziehung gesetzt werden. Eine Reihe von interessanten Fragen wird durch die Zusammenführung von Informationen aus den Befragungen und aus den anderen Teilen des Surveys (körperliche Untersuchung, Laboruntersuchungen) beantwortet werden können. Einige Angaben aus den Befragungen können auf diese Art und Weise ergänzt, überprüft bzw. validiert werden. Beispiele sind die Angaben zu Infektionskrankheiten und zu Impfungen und die Antikörperwerte im Serum; die Angaben zu Sehstörungen und die Ergebnisse des Sehtests; oder die Angaben zu Schilddrüsenerkrankungen, die Erfassung der Schilddrüsenmedikamente, die gemessenen Schilddrüsenhormone im Serum und die durchgeführte Schilddrüsensonographie.

\section{Erfahrungen aus dem Pretest}

Ziel des Pretests war es, die Befragungsinstrumente zu prüfen und zu verbessern. Methodisch war vor allem von Interesse, ob 
Fragenformulierungen und Antwortkategorien eindeutig, gut verständlich und im Hinblick auf die Auswertung sinnvoll sind, ob sie noch weiter standardisiert werden können und ob die Verteilung der Fragen auf das ärztliche Interview und die Fragebogen sinnvoll und angemessen ist. Weitere wichtige Parameter waren die Akzeptanz durch die Probanden, die Befragungsdauer und das Zusammenspiel mit den anderen Surveyteilen.

Im Rahmen des Pretests wurden Fragebogen für 1626 Kinder und Jugendliche ausgefüllt und ärztliche Interviews mit den Eltern von 1571 Kindern und Jugendlichen durchgeführt. Generell war die Akzeptanz für die Fragen zur körperlichen Gesundheit in den Fragebogen und im ärztlichen Interview sehr gut. Die Erfahrungen des Pretests wurden dazu genutzt, Fragenformulierungen und Antwortkategorien zu präzisieren. Die offenen Fragen mit Freitextangaben in den Fragebogen und im ärztlichen Interview wurden ausgewertet und dazu verwendet, die Relevanz und die Priorität jeder einzelnen Frage nochmals kritisch zu beleuchten und das Spektrum der abgefragten Krankheiten weiter zu optimieren. Je nach Spektrum der relevanten Erkrankungen variieren die Befragungsinstrumente in den fünf Altersstufen leicht. Die erfassten Prävalenzen von Erkrankungen wurden dort, wo Vergleichswerte aus der Literatur existieren - auf ihre Plausibilität hin geprüft, und es wurden ggf. Rückschlüsse auf die Befragungsmethodik gezogen.

Im Unterschied zur Hauptphase wurden im Pretest alle Fragen zu chronischen Krankheiten sowohl in den Fragebogen als auch im ärztlichen Interview gestellt. Die Fragebogen lagen der interviewenden Ärztin vor, so dass Angaben hinterfragt und Gründe für Missverständnisse dokumentiert werden konnten. Auf der Basis dieser Erfahrung erscheint es für die meisten chronischen Erkrankungen sinnvoller, sie im ärztlichen Interview abzufragen. Die Abfrage von ärztlichen Diagnosen stellt - in gewissen Grenzen - eine einheitliche Definition der Erkrankungen sicher und spiegelt zugleich das Diagnosenspektrum, das dem Versorgungsgeschehen zugrunde liegt. Zusätzlich zeigte der Pretest, dass Plausibilitätsprüfungen und begriffliche Klärungen durch die Studienärztin oft Missverständnisse zutage brachten und präzisere Angaben ermöglichten. Eine Ausnahme bildet das allergische Kontaktekzem: Es würde bei Erfassung als ärztliche Diagnose zu einer falsch niedrigen Prävalenz führen, weil viele Kinder und Jugendliche sich damit nicht ärztlich vorstellen. Es wird aber fast immer von Eltern oder Jugendlichen richtig erkannt und begrifflich eingeordnet, so dass eine Fragebogenfrage valide erscheint. Eine Doppelabfrage, die auch Differenzen zwischen ärztlichen und eigenen Diagnosen aufzeigt, muss aufgrund der Fragebogen- und Interviewlänge auf einige wenige chronische Krankheiten wie Heuschnupfen und Neurodermitis beschränkt werden.

Interessant - und letztlich nicht unerwartet - war, dass Eltern und Jugendliche identische Fragen zu Krankheiten z.T. unterschiedlich beantworten. Die Gründe hierfür variieren von Frage zu Frage. Im Pretest lagen der Ärztin die Krankheitsangaben der Jugendlichen und der Eltern aus dem Selbstausfüllfragebogen vor. Sie konnten daher mit den Eltern und Jugendlichen besprochen werden. Es zeigte sich, dass Erkrankungen z.T. unterschiedlich erinnert, Arztdiagnosen verschieden wahrgenommen oder verstanden werden, Beschwerden anders interpretiert werden.
Meist ist nicht pauschal zu entscheiden, ob die Eltern oder die Kinder und Jugendlichen validere Antworten geben. In der Hauptphase sollen daher möglichst viele Fragen zur körperlichen Gesundheit sowohl den Eltern als auch den Kindern und Jugendlichen gestellt werden. Unterschiede zwischen den Angaben werden insbesondere bei den Fragen zu subjektiven Aspekten der körperlichen Gesundheit, nämlich Beschwerden und Schmerzen, dem körperlichen Wohlbefinden und der allgemeinen Einschätzung der Gesundheit interessant sein.

\section{Literatur}

${ }^{1}$ Barker DJ, Gluckman PD, Godfrey KM et al. Fetal nutrition and cardiovascular disease in adult life. Lancet 1993; 341: 938-941

2 Beasley R, Crane J, Lai CK et al. Prevalence and etiology of asthma. J Allergy Clin Immunol 2000; 105: 466-472

${ }^{3}$ Bethell CD, Read D, Stein RE et al. Identifying children with special health care needs: development and evaluation of a short screening instrument. Ambul Pediatr 2002; 2: 38-48

${ }^{4}$ Blake KV, Gurrin LC, Evans SF et al. Adjustment for current weight and the relationship between birth weight and blood pressure in childhood. J Hypertens 2000; 18: 1007-1012

${ }^{5}$ Bundeszentrale für gesundheitliche Aufklärung (Hrsg). Gesundheit von Kindern - Epidemiologische Grundlagen. Expertentagung. Datenquellen, Datenqualität und Gesundheitsberichte. Köln 1998; 16-46

${ }^{6}$ Currie C, Hurrelmann K, Settertobulte W et al. Health and Health Behaviour among young People. Health Behaviour in School-aged Children: a WHO Cross-National Study (HBSC). International Report. Health Policy for Children and Adolescents (HEPCA): WHO Regional Office for Europe 2000; Series No. 1: 26-35

${ }^{7}$ De Bruin A, Picavet HSJ, Nossikov A. Health Interview Surveys. Towards harmonization of methods and instruments. WHO Regional Publications. Copenhagen 1996; European Series No. 58: 51-53

${ }^{8}$ Dörner G, Plagemann A. Perinatal hyperinsulinism as possible predisposing factor for diabetes mellitus, obesity and enhanced cardiovascular risk in later life. Horm Metab Res 1994; 26: 213-221

${ }^{9}$ Gillman MW. Epidemiological challenges in studying the fetal origins of adult chronic disease. Int J Epidemiol 2002; 31: 294-299

${ }^{10}$ Goodman R. Psychometric properies of the strengths and difficulties questionnaire. J Am Acad Child Adolesc Psychiatry 2001; 40: $1337-1345$

${ }^{11}$ Gordon T. Three views of hypertension and heart disease. National Center for Health Statistics. Vital Health Stat 1967; 2 (22): 1-43

${ }^{12}$ Hoepner-Stamos F. Prävalenz und Ätiologie chronischer Erkrankungen im Kindes- und Jugendalter. In: Kolip P, Hurrelmann K, Schnabel PE (Hrsg). Jugend und Gesundheit. Weinheim: Juventa 1995; 49-67

${ }^{13}$ Idler EL, Benyamini Y. Self-rated health and mortality: a review of twenty-seven community studies. J Health Soc Behav 1997; 38: 21-37

${ }^{14}$ Kaplan GA, Goldberg DE, Everson SA et al. Perceived health status and morbidity and mortality: evidence from the Kuopio ischaemic heart disease risk factor study. Int J Epidemiol 1996; 25: 259-265

${ }^{15}$ Knopf H, Bergmann E, Dippelhofer A et al. Der Kinder- und Jugendgesundheitssurvey als Datenquelle zur Beschreibung wesentlicher Aspekte der gesundheitlichen Versorgung im Kindes- und Jugendalter. Gesundheitswesen 2002; 64 (Sonderheft 1): 43-47

${ }^{16}$ Kolip P, Nordlohne E, Hurrelmann K. Der Jugendgesundheitssurvey 1993. In: Kolip P, Hurrelmann K, Schnabel PE (Hrsg). Jugend und Gesundheit. Juventa: Weinheim 1995; 25-48

${ }^{17}$ Kuh D, Ben-Shlomo Y (Hrsg). A Life Course Approach to Chronic Disease Epidemiology. Oxford/New York/Tokyo: Oxford University Press 1997

${ }^{18}$ Miller JE, Gaboda D, Davis D. Early childhood chronic illness: comparability of maternal reports and medical records. National Center for Health Statistics. Vital Health Stat 2001; 2 (131): 1-10

${ }^{19}$ Newacheck PW, Taylor WR. Childhood chronic illness: prevalence, severity and impact. Am J Public Health 1992; 82: 364-371

${ }^{20}$ Newascheck PW, Halfon N. Prevalence, impact, and trends in childhood disability due to asthma. Arch Pediatr Adolesc Med 2000; 154: 287-293

${ }^{21}$ Perquin CW, Hazebroek-Kampschreur AA, Hunfeld JA et al. Pain in children and adolescents: a common experience. Pain 2000 (a); 87: 51-58 
22 Perquin CW, Hazebroek-Kampschreur AA, Hunfeld JA et al. Chronic pain among children and adolescents: physician consultation and medication use. Clin J Pain 2000 (b); 16: 229-235

${ }^{23}$ Ravens-Sieberer U, Bullinger M. Assessing health-related quality of life in chronically ill children with the German KINDL: first psychometric and content analytical results. Qual Life Res 1998; 7: 399-407

${ }^{24}$ Roth-Isigkeit A, Ellert U, Kurth BM. Die Erfassung von Schmerz in einem Kinder- und Jugendgesundheitssurvey. Gesundheitswesen 2002; 64 (Sonderheft 1): 125-129

${ }^{25}$ Silink M. Childhood diabetes: a global perspective. Horm Res 2002; 57 (Suppl 1): 1-5

${ }^{26}$ Statistisches Bundesamt. Fragen zur Gesundheit 1982. Mainz: Kohlhammer 1984; Fachserie 12, Reihe S.3: 10
${ }^{27}$ The European Health Report 2002. WHO regional publications. Copenhagen 2002; European series; No. 97: 52

28 Whitaker RC, Dietz WH. Role of the prenatal environment in the development of obesity. J Pediatr 1998; 132: 768-776

${ }^{29}$ Zill N. Advantages and limitations of using children and adolescents as survey respondents. In: Cynamon ML, Kulka RA (Hrsg). Seventh conference on health survery research methods. Department of Health and Human Services, Centers for Disease Control and Prevention: National Center for Health Statistics. Hyattsville, Maryland 2001; DHHS Publication No. (PHS) 01-1013: 47-50 